\title{
Generation of three-port splitter by double-layer grating in second-order Littrow configuration
}

\author{
Chenhao Gao, Bo Wang ${ }^{*}$, Chen Fu, Jimin Fang, Kunhua Wen, Ziming Meng, \\ Zhaogang Nie, Xianguun Xing, Li Chen, Liang Lei, Jinyun Zhou
}

School of Physics and Optoelectronic Engineering, Guangdong University of Technology, Guangzhou 510006, China

*Corresponding author: wangb_wsx@yeah.net

\begin{abstract}
In this paper, a novel double-layer three-port grating is described. The incident grating structure is in the second-order Littrow configuration. The grating region is composed of fused silica and $\mathrm{Ta}_{2} \mathrm{O}_{5}$. The designed grating beam splitter has high efficiency under TE polarization and TM polarization, respectively. The efficiency of two polarizations is more than $90 \%$. In addition, compared with a single-layer three-port grating, this new beam splitter has good fabrication tolerance and incident bandwidth. Therefore, the optimized structure has a good application value.
\end{abstract}

Keywords: three-port splitter, second-order Littrow, double-layer grating.

\section{Introduction}

Grating is a common optical device, which can perform different functions depending on the fabricated material and structure, such as polarization, splitting, filtering, etc. [1- $\underline{1}$ ]. The grating can be designed as an anti-reflector [ $[\underline{6}$, a phase retardation waveplate [ $\underline{7}]$, a polarization beam splitter [ $\underline{8}$ ], and a wavelength selective reflection filter [9] according to different functions. For example, fused-silica grating $[\underline{10}, 11]$ has good thermal stability and high damage threshold. And the polarizing beam splitter [12-16] etched in fused silica has good diffraction efficiency and high utilization. Moreover, it has important applications in the fields of lithography, laser pulses, spectroscopic, and interferometers. ZHENG et al. [17] generally proposed a single-layer three-port grating under TE polarization. The diffraction efficiencies in the -2 nd order, -1 st order and 0 th order were $28.84 \%, 28.85 \%$, and $28.85 \%$, respectively [17]. The overall efficiency is $86.54 \%$. The incident light is under the second Bragg grating structure mode. Different from the single-layer grating, the grating thickness of the two layers affects the beam splitting effect of the grating. We propose a two-layer three-port fused-silica grating, which is much more efficient than reported in Ref. [17]. The total efficiency of our design has 
increased to $11.4 \%$ compared with Ref. [17] under TE polarization. Moreover, TM polarization is also considered in this design. While in Ref. [17], only TE polarization is optimized for the three-port splitting. For practical production, the influence of technological tolerance on grating is investigated in this design.

Based on the theoretical analysis of gratings, we mainly use the vector diffraction theory [18]. In this design, we mainly use the rigorous coupled-wave analysis (RCWA) [19]. This method has been improved continuously and can deal with grating of different plane and medium. Different from the model method [20], the strictly coupled wave is expanded according to different diffraction orders, and then the diffraction field is obtained by the boundary conditions. RCWA is a direct and effective electromagnetic field theory. It solves the problem of solving Maxwell's equation strictly in the grating region. The electromagnetic field expression of the grating region coupled by the eigenfunction is obtained. The value of the final diffraction efficiency is obtained by solving the boundary conditions at the interface. The diffraction phenomenon of grating cannot be explained by a strictly coupled wave. The mode method can be used to describe the working mechanism of grating more simply and intuitively.

In this paper, a novel three-port beam splitter based on double-layer fused-silica grating is designed. We get better numerical results by rigorous coupled-wave optimization. For TE polarization, the diffraction efficiency of the three diffraction orders was $97.94 \%$ overall. For TM polarization, the diffraction efficiency of the three diffraction orders is $98.08 \%$. We analyze the manufacturing tolerance and incident characteristics of the grating. The grating beam splitter has a large period tolerance and good angular bandwidth for TE polarization. High-efficiency beam splitter is well suited for use in the laser field.

\section{Optimization and modal analysis of beam splitter}

The designed beam splitter is shown in Fig. 1. For this double-layer grating structure, the original grating period can be reduced compared with the conventional single-layer three-port transmission grating. For ease of design and production, the duty cycle $f$ is set to 0.5 and the period $d$ is designed to be $953 \mathrm{~nm}$ by calculation. The incident wavelength is $800 \mathrm{~nm}$. The angle of incidence is $\theta=\sin ^{-1}(\lambda / d)$. The incident light is incident under the second Bragg Littrow mounting. The grating substrate is made of fused-silica medium. The first layer is made of $\mathrm{Ta}_{2} \mathrm{O}_{5}$ with a depth of $h_{1}$ and a refractive index of $n_{3}=2.00$. The second layer is made of the same material as the substrate with a depth of $h_{2}$, and the refractive index is $n_{2}=1.45$. TE polarization is the incident light whose electric field is parallel to the direction of the grating groove. TM polarization is that the direction of the electric field is perpendicular to the grating groove. The three-port splitter can be designed for TE or TM polarization by double-layer grating in the second-order Littrow configuration. After the incident light is split by the grating, most of the energy can only be split to the 0 th, -1 st and -2 nd order. The grating parameters and 


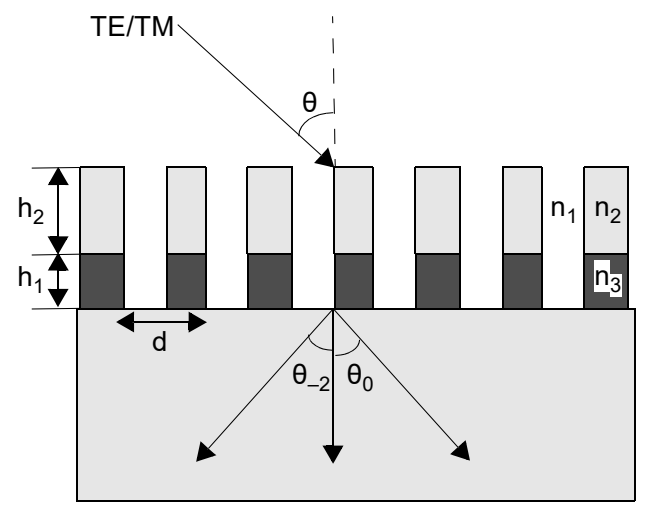

Fig. 1. The schematic diagram of the double-layer three-port rectangular grating under second Bragg incidence.

incident conditions are the factors that affect the performance of the grating. The grating parameters and incident conditions are factors that affect the performance of the grating, including incident angle, duty cycle, period, and groove depths. The incident angle is under the second Bragg Littrow mounting. In this design, in order to concentrate the energy on the specific diffraction order of the grating, we first design the corresponding grating period and duty cycle. Then, the accurate depths of two layers can be optimized by RCWA.

For the optimization, Fig. 2 shows the relationship between the depth of two layers and efficiency of the grating under two polarizations. The known duty cycle is 0.5 and
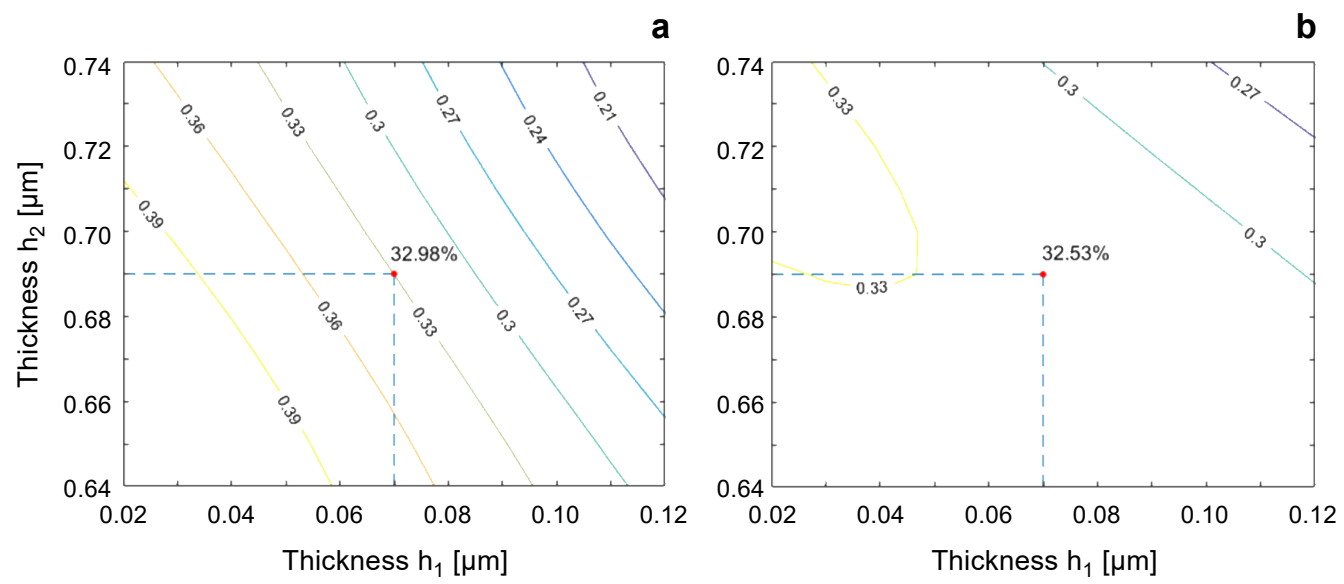

Fig. 2. Efficiencies in three orders for the grating versus depth of two layers at wavelength of $800 \mathrm{~nm}$ with duty cycle of 0.5 under second Bragg incidence: the 0 th order for TE polarization (a), the -1 st order for TE polarization (b), the -2 nd order for TE polarization (c), the 0th order for TM polarization (d), the -1 st order for TM polarization (e), and the -2 nd order for TM polarization (f). 

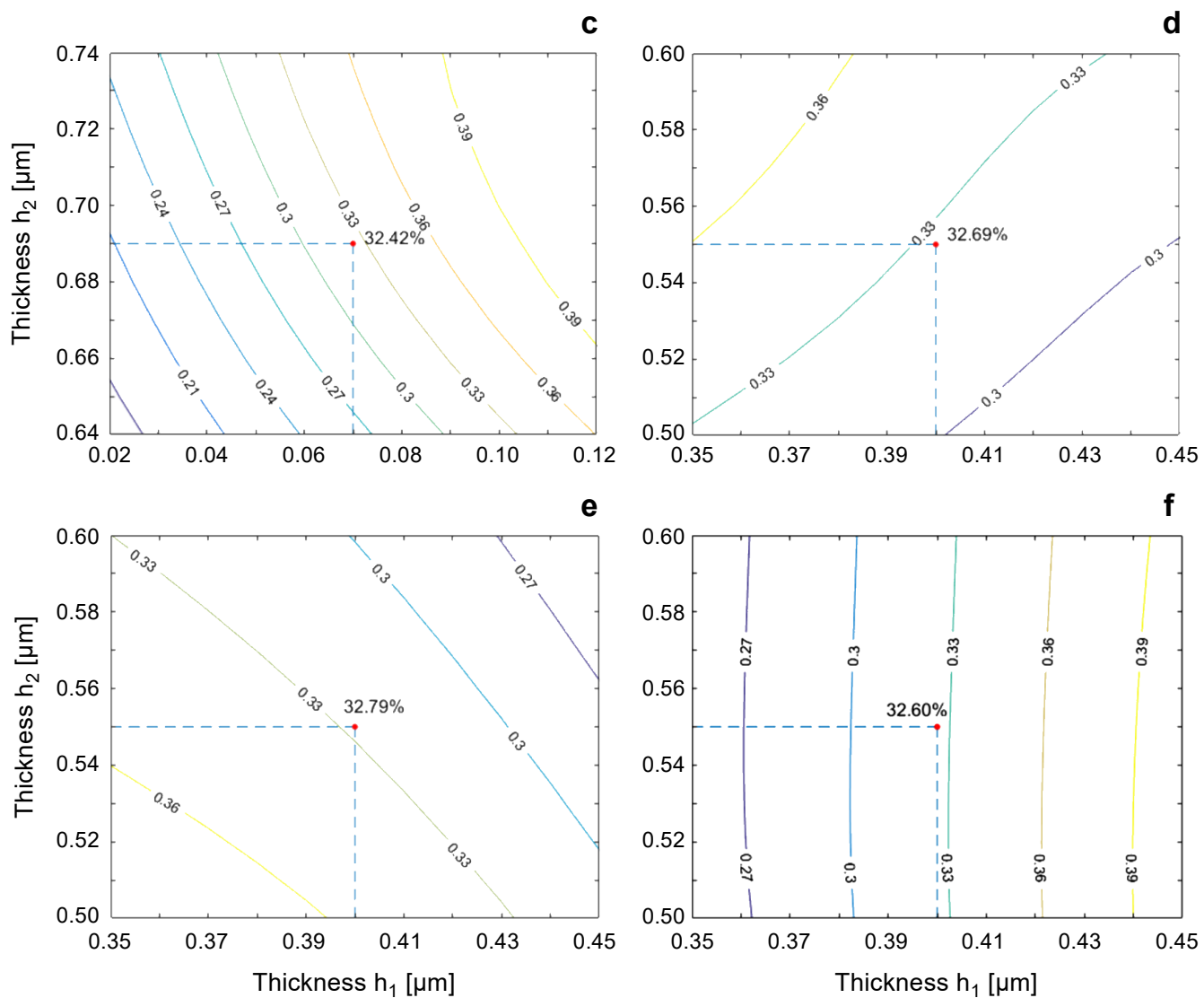

Fig. 2. Continued.

the period is $953 \mathrm{~nm}$. After the optimization of the rigorous coupled wave, the following higher parameters are obtained. For TE polarization, the optimized parameters of the grating are $h_{1}=0.07 \mu \mathrm{m}$ and $h_{2}=0.69 \mu \mathrm{m}$. At this time, the efficiency of the 0 th order is $32.98 \%$, the efficiency of the -1 st order is $32.53 \%$, and the efficiency of the -2 nd order is $32.42 \%$. The ratio of the -1 st to the 0 th order efficiency for TE polarization is 0.9863 , and the ratio of the -2 nd to the -1 st order for TE polarization is 0.9966 . For TM polarization, the optimized parameters of the grating are $h_{1}=0.4 \mu \mathrm{m}$ and $h_{2}=0.55 \mu \mathrm{m}$. At this time, the efficiency of the 0 th order is $32.69 \%$, the efficiency of the -1 st order is $32.79 \%$, and the efficiency of the -2 nd order is $32.60 \%$. The ratio of the -1 st to the 0 th order efficiency is 1.003 , and the ratio of the -2 nd to -1 st order is 0.9942 .

Based on the modal method, Fig. 3 reflects the relationship between the effective refractive index and the period when the incident wavelength of the grating is $800 \mathrm{~nm}$, duty cycle is 0.5 under the second Bragg angle incidence. The effective refractive index of the grating is related to the period of the grating and the refractive index of each layer of the grating. The mode coupling and diffraction efficiency of the grating are 


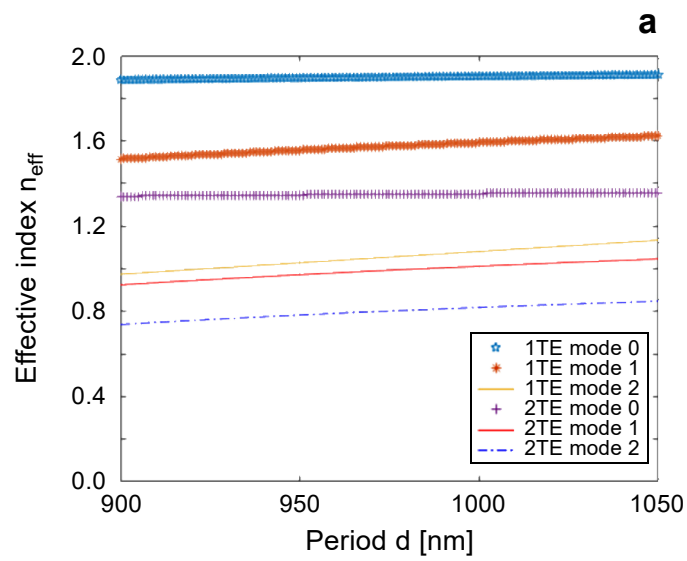

b

Fig. 3. Effective index versus the grating period with duty cycle of 0.5 and wavelength of $800 \mathrm{~nm}$ under second Bragg incidence: TE polarization (a), and TM polarization (b).

T a b l e. The effective refractive index of double-layer grating.

\begin{tabular}{lllll}
\hline & \multicolumn{2}{l}{ The first layer } & \multicolumn{2}{l}{ The second layer } \\
\hline Polarization & TE & TM & TE & TM \\
\hline Mode 0 & 1.8957 & 1.8446 & 1.3470 & 1.3023 \\
Mode 1 & 1.5589 & 1.3427 & 0.9746 & 0.8714 \\
Mode 2 & 1.0313 & 1.0103 & 0.7851 & 0.8526 \\
\hline
\end{tabular}

related to the effective refractive index of the double-layer medium. The effective refractive index is determined by solving the dispersion equations [ $\underline{21}-\underline{23}]$ for TE polarization

$$
\begin{aligned}
F\left(n_{\text {eff }}^{2}\right) & =\cos \left(k_{1}(1-f) d\right) \cos \left(k_{2} f d\right)-\frac{k_{1}^{2}+k_{2}^{2}}{2 k_{1} k_{2}} \sin \left(k_{1}(1-f) d\right) \sin \left(k_{2} f d\right) \\
& =\cos (\alpha d)
\end{aligned}
$$

and for TM polarization

$$
\begin{aligned}
F\left(n_{\mathrm{eff}}^{2}\right) & =\cos \left(k_{1}(1-f) d\right) \cos \left(k_{2} f d\right)-\frac{n_{2}^{4} k_{1}^{2}+k_{2}^{2}}{2 n_{2}^{2} k_{1} k_{2}} \sin \left(k_{1}(1-f) d\right) \sin \left(k_{2} f d\right) \\
& =\cos (\alpha d)
\end{aligned}
$$

where $k_{m}=k_{0} \sqrt{n_{m}^{2}-n_{\text {eff }}^{2}}, \alpha=k_{0} \sin \theta$, and $k_{0}=2 \pi / \lambda$.

The effective refractive index of grating can be calculated by the mode method, which is shown in the Table. Therefore, the propagating constants of two layers for TE-polarized light are: 


$$
\begin{aligned}
& \delta_{0}^{1 \mathrm{TE}}=\frac{2 \pi}{\lambda} n_{0, \text { eff }}^{1 \mathrm{TE}}=1.4881 \times 10^{7} \mathrm{~m}^{-1} \\
& \delta_{1}^{1 \mathrm{TE}}=\frac{2 \pi}{\lambda} n_{1, \text { eff }}^{1 \mathrm{TE}}=1.2237 \times 10^{7} \mathrm{~m}^{-1} \\
& \delta_{2}^{1 \mathrm{TE}}=\frac{2 \pi}{\lambda} n_{2, \text { eff }}^{1 \mathrm{TE}}=8.0957 \times 10^{6} \mathrm{~m}^{-1} \\
& \delta_{0}^{2 \mathrm{TE}}=\frac{2 \pi}{\lambda} n_{0, \text { eff }}^{2 \mathrm{TE}}=1.0573 \times 10^{7} \mathrm{~m}^{-1} \\
& \delta_{1}^{2 \mathrm{TE}}=\frac{2 \pi}{\lambda} n_{1, \mathrm{eff}}^{2 \mathrm{TE}}=7.6506 \times 10^{6} \mathrm{~m}^{-1} \\
& \delta_{2}^{2 \mathrm{TE}}=\frac{2 \pi}{\lambda} n_{2, \mathrm{eff}}^{2 \mathrm{TE}}=6.1630 \times 10^{6} \mathrm{~m}^{-1}
\end{aligned}
$$

For TM-polarized light, the propagating constants can be calculated and shown that:

$$
\begin{aligned}
& \delta_{0}^{1 \mathrm{TM}}=\frac{2 \pi}{\lambda} n_{0, \text { eff }}^{1 \mathrm{TM}}=1.4480 \times 10^{7} \mathrm{~m}^{-1} \\
& \delta_{1}^{1 \mathrm{TM}}=\frac{2 \pi}{\lambda} n_{1, \text { eff }}^{1 \mathrm{TM}}=1.0540 \times 10^{7} \mathrm{~m}^{-1} \\
& \delta_{2}^{1 \mathrm{TM}}=\frac{2 \pi}{\lambda} n_{2, \text { eff }}^{1 \mathrm{TM}}=7.9308 \times 10^{6} \mathrm{~m}^{-1} \\
& \delta_{0}^{2 \mathrm{TM}}=\frac{2 \pi}{\lambda} n_{0, \text { eff }}^{2 \mathrm{TM}}=8.2998 \times 10^{6} \mathrm{~m}^{-1} \\
& \delta_{1}^{2 \mathrm{TM}}=\frac{2 \pi}{\lambda} n_{1, \text { eff }}^{2 \mathrm{TM}}=6.8404 \times 10^{6} \mathrm{~m}^{-1} \\
& \delta_{2}^{2 \mathrm{TM}}=\frac{2 \pi}{\lambda} n_{2, \mathrm{eff}}^{2 \mathrm{TM}}=6.6929 \times 10^{6} \mathrm{~m}^{-1}
\end{aligned}
$$

The modal method can be used to explain the diffraction behavior of light waves in the grating. When light is incident from the upper surface of the grating to the lower surface, two couplings occur. The first coupling is that the light enters the grating, where three grating modes are excited: mode 0 , mode 1 and mode 2 . Three modes have different effective refractive indices and propagate with different propagation constants. The energy exchange exists between the incident wave and the grating mode [24]. The second coupling occurs between the grating mode and the diffraction order. Through energy exchange for two layers, the physical explanation can be illustrated for the improved three-port splitting grating. 


\section{Manufacturing tolerance and investigation of incident performance}

Considering practical manufacture, Fig. 4 shows the relationship between period tolerance and efficiency under the second Bragg angle. Compared to the other three-port beam splitter, the grating has a high period fabrication tolerance. This is the performance that needs to be considered in actual production. As shown in Fig. 4a, the efficiency of the grating is greater than $30 \%$ in the range of $922-1016 \mathrm{~nm}$ for TE polarization. As shown in Fig. $4 \mathbf{b}$, the efficiency is greater than $30 \%$ in the range of $940-964 \mathrm{~nm}$ for TM polarization. The data shows that the grating period tolerance under TE polarization is high. The incident wavelength bandwidth and angular bandwidth are also parameters for studying the grating beam splitting structure. In the optimization process, the incident wave is always under the second Bragg incident angle.
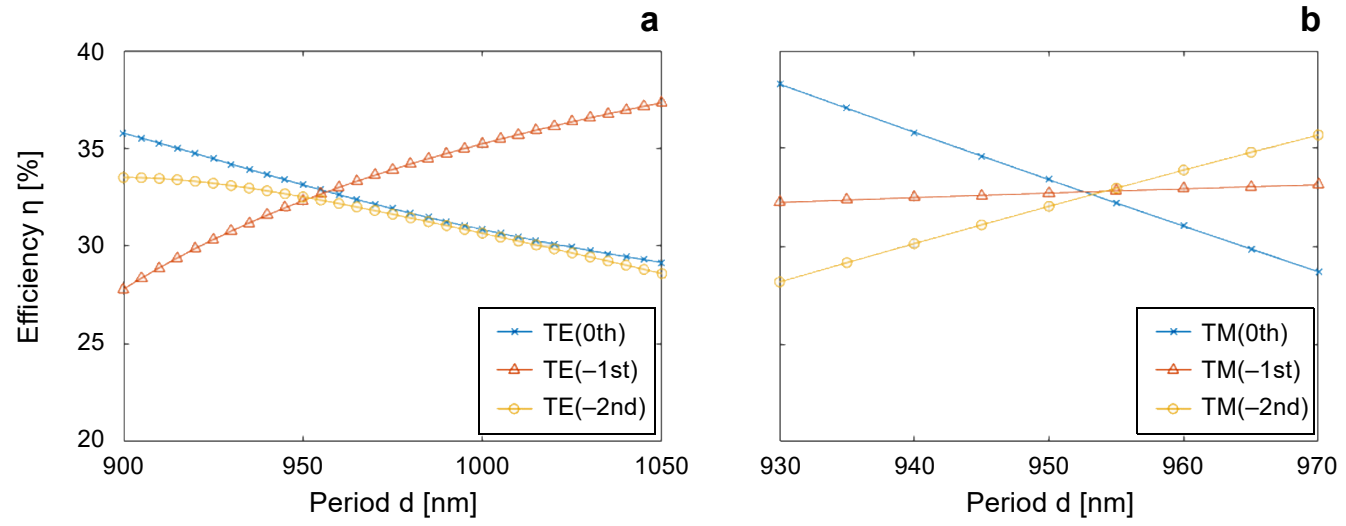

Fig. 4. The efficiency corresponding to the period for TE polarization (a), and TM polarization (b).
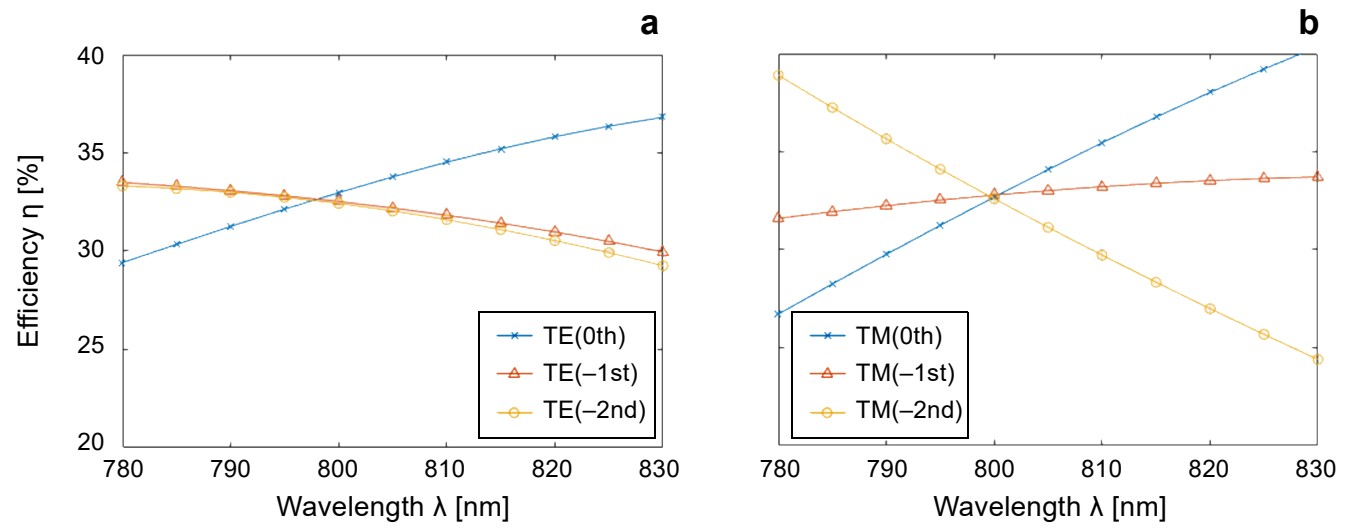

Fig. 5. The efficiency corresponding to the incident wavelength for TE polarization (a), and TM polarization (b). 
a

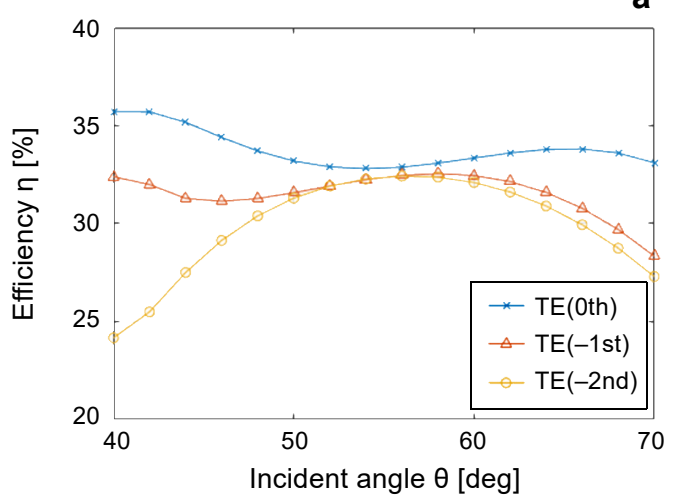

b

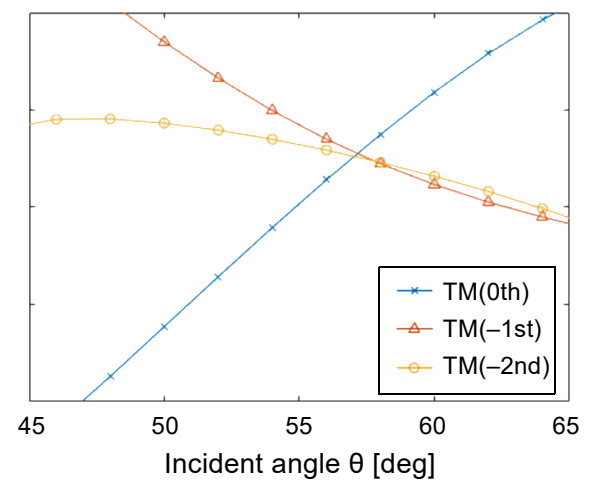

Fig. 6. The diffraction efficiency corresponding to the incident second Bragg angle: TE polarization (a), and TM polarization $(\mathbf{b})$.

Figure 5 reflects the relationship between the efficiency of the grating stages and the incident wavelength. As shown in Fig. 5, for TE polarization, the wavelength bandwidth of greater than $30 \%$ is in the range of $784-824 \mathrm{~nm}$ and the bandwidth is $40 \mathrm{~nm}$. For TM polarization, the wavelength bandwidth of greater than $30 \%$ is $791-809 \mathrm{~nm}$ and the bandwidth is $18 \mathrm{~nm}$. Figure 6 reflects the relationship between the incident angle and the efficiency of three diffraction orders. Under TE polarization, the angular efficiency of the grating efficiency is greater than $30 \%$ and the angular bandwidth is 47.3-65.9 deg. The bandwidth is $18.6 \mathrm{deg}$. Under TM polarization, the grating efficiency is greater than $30 \%$ for the angular bandwidth range from 54.9 to 62.6 deg with a bandwidth of $7.7 \mathrm{deg}$.

\section{Conclusions}

In order to improve the efficiency and incident characteristics of three-channel gratings, the grating structure is optimized by rigorous coupled-waves. $\mathrm{A} \mathrm{SiO}_{2} / \mathrm{Ta}_{2} \mathrm{O}_{5}$ double-layer rectangular grating with second Bragg incidence is proposed in this paper. The transmittance of TE-polarized light can reach $97.93 \%$, while that of TM-polarized light can reach $98.08 \%$. Compared with single-layer three-port grating, the design also has a higher bandwidth of incident wavelength and a wide range of incident angles. The double-layer thickness and period of the grating are relatively small, which is beneficial to the fabrication and application of the grating.

Acknowledgements - This work is supported by the Science and Technology Program of Guangzhou (202002030284, 202007010001).

\section{References}

[1] Zhibin Ren, Yahui Sun, Zihao Lin, Chunyu Wang, Wanying Huang, Tunable nonpolarizing guided-mode resonance filter incorporating electrically driving liquid crystal, Infrared Physics and Technology 95, 2018, pp. 39-45, DOI: 10.1016/j.infrared.2018.10.016. 
[2] Jie Wang, Hongdan Wan, Han Cao, Yu Cai, Bing Sun, Zuxing Zhang, Lin Zhang, a 1- $\mu m$ cylindrical vector beam fiber ring laser based on a mode selective coupler, IEEE Photonics Technology Letters 30(9), 2018, pp. 765-768, DOI: 10.1109/LPT.2018.2797990.

[3] Dehdast M., Bahrami A., Mohammadnejad S., A novel trapezoidal profile of optimized diffraction grating for light trapping in thin silicon solar cells, Optica Applicata 47(1), 2017, pp. 75-83, DOI: $10.5277 / 0 a 170107$.

[4] Asgari N., Hamidi S.M., Fantastic exciton-plasmon coupling in dye-doped poly (vinyl pyrrolidone)/ gold one-dimensional nano-grating, Superlattices and Microstructures 123, 2018, pp. 358-373, DOI: 10.1016/j.spmi.2018.09.019.

[5] Yao Zhu, Congying Feng, Yuze Wu, Yonglin Yu, A chirp-managed tunable DBR laser based on novel cascaded gratings, IEEE Photonics Journal 10(5), 2018, article 7203810, DOI: 10.1109/ JPHOT.2018.2871513.

[6] Yousefi P., Schonenberger N., Mcneur J., Kozak M., Niedermayer U., Hommelhoff P., Dielectric laser electron acceleration in a dual pillar grating with a distributed Bragg reflector, Optics Letters 44(6), 2019, pp. 1520-1523, DOI: 10.1364/OL.44.001520.

[7] Banghong Zhang, Yandong Gong, Achromatic terahertz quarter waveplate based on silicon grating, Optics Express 23(11), 2015, pp. 14897-14902, DOI: 10.1364/OE.23.014897.

[8] Wenjing Fang, Xinye Fan, Xia Zhang, Huijuan Niu, Hengying Xu, Jiarui Fei, Yongqing Huang, ChENGLIN BAI, Polarization-insensitive high-index contrast gratings beam splitter with focusing ability, IEEE Photonics Technology Letters 30(8), 2018, pp. 708-711, DOI: 10.1109/LPT.2018.2812866.

[9] Jun Gu, Fei Wang, Youxi Lu, Mengmeng Peng, Lun Shi, Wen Kang, Jingxin Huang, Polarization dependence of stimulated Brillouin scattering-based switchable microwave photonic filter, Optica Applicata 49(1), 2019, pp. 5-11, DOI: 10.5277/oa190101.

[10] VoznYuk L., Boutami S., Gliere A., Enlarging the bandwidth of a two-dimensional photonic crystal mirror in the visible range, Optica Applicata 47(2), 2017, pp. 175-182, DOI: 10.5277/oa170201.

[11] Man Jiang, Pu Zhou, XiJia Gu, Ultralong $\pi$-phase shift fiber Bragg grating empowered single-longitudinal mode DFB phosphate fiber laser with low-threshold and high-efficiency, Scientific Reports 8, 2018, article 13131, DOI: 10.1038/s41598-018-31528-w.

[12] Weimin Wang, Xufeng Jing, Jingyin Zhao, Yinyan Li, Ying Tian, Improvement of accuracy of simple methods for design and analysis of a blazed phase grating microstructure, Optica Applicata 47(2), 2017, pp. 183-198, DOI: 10.5277/oa170202.

[13] Yingxuan Zhao, Chao Qiu, Aimin Wu, Haiyang Huang, Jun Li, Zhen Sheng, Wei Li, Xi Wang, FUWAN GAN, Broadband polarization splitter-rotator and the application in WDM receiver, IEEE Photonics Journal 11(1), 2019, article 6600310, DOI: 10.1109/JPHOT.2018.2886268.

[14] JinBIAO XIAO, ZHENZHAO GUO, Ultracompact polarization-insensitive power splitter using subwavelength gratings, IEEE Photonics Technology Letters 30(6), 2018, pp. 529-532, DOI: 10.1109/LPT. 2018.2801337.

[15] Yifan Tang, Yifan Zhu, Bin Liang, Jing Yang, Jun Yang, Jianchun Cheng, One-way acoustic beam splitter, Scientific Reports 8, 2018, article 13573, DOI: 10.1038/s41598-018-29579-0.

[16] Xuan Zhao, Yihang Li, Shuai Feng, Xiao Chen, Chuanbo Li, Yiquan Wang, Beam splitting characteristics of two-dimensional photonic crystals based on surface modulation, Optics Communications 439, 2019, pp. 193-200, DOI: 10.1016/j.optcom.2019.01.021.

[17] Jianguun Zheng, Changhe Zhou, Bo Wang, Jijun Feng, Beam splitting of low-contrast binary gratings under second Bragg angle incidence, Journal of the Optical Society of America A 25(5), 2008, pp. 1075-1083, DOI: 10.1364/JOSAA.25.001075.

[18] Hao Huang, Tingting Zhai, Qiang Song, Xiaodong Yin, Wide angle 2D beam splitter design based on vector diffraction theory, Optics Communications 434, 2019, pp. 28-35, DOI: 10.1016/j.optcom. 2018.10.026.

[19] Moharam M.G., Pommet D.A., Grann E.B., GaYlord T.K., Stable implementation of the rigorous coupled-wave analysis for surface-relief gratings: enhanced transmittance matrix approach, Journal of the Optical Society of America A 12(5), 1995, pp. 1077-1086, DOI: 10.1364/JOSAA.12.001077. 
[20] Botten I.C., Craig M.S., McPhedran R.C., Adams J.L., Andrewartha J.R., The dielectric lamellar diffraction grating, Optica Acta 28(3), 1981, pp. 413-428, DOI: 10.1080/713820571.

[21] Ahmed F., Ahsani V., Jo S., Bradley C., Toyserkani E., Jun Martin B.G., Measurement of in-fiber refractive index change using a Mach-Zehnder interferometer, IEEE Photonics Technology Letters 31(1), 2019, pp. 74-77, DOI: 10.1109/LPT.2018.2883913.

[22] Xiao Wang, Bin Lu, Zhaoyong Wang, Hanrong Zheng, Jiajing Liang, Luchuan Li, Qing Ye, Ronghui Qu, HaIwEN CAI, Interference-fading-free $\Phi$-OTDR based on differential phase shift pulsing technology, IEEE Photonics Technology Letters 31(1), 2019, pp. 39-42, DOI: 10.1109 / LPT.2018.2881757.

[23] Xufeng Jing, Rui Xia, Xincui Gui, Weimin Wang, Ying Tian, Dongshuo Zhu, Guohua Shi, Design of ultrahigh refractive index metamaterials in the terahertz regime, Superlattices and Microstructures 109, 2017, pp. 716-724, DOI: 10.1016/j.spmi.2017.05.061.

[24] Hongtao Li, Bo Wang, Hao Pei, Li Chen, Liang Lei, Jinyun Zhou, Fused-silica sandwiched three -port grating under second Bragg angle incidence, Superlattices and Microstructures 93, 2016, pp. 157-162, DOI: $\underline{10.1016 / j . s p m i .2016 .03 .027 . ~}$

Received May 16, 2019

in revised form July 3, 2019 